\title{
A Conformal Multi-Frequency Antenna Array for Safety-of-Life Satellite Navigation
}

\author{
Kazeem A. Yinusa, Lukasz A. Greda and Achim Dreher \\ German Aerospace Center (DLR) \\ Institute of Communications and Navigation \\ Oberpfaffenhofen, Wessling 82234, Germany \\ Email: kazeem.yinusa@dlr.de
}

\begin{abstract}
Satellite navigation is finding new applications in safety critical systems such as in aviation where it is proposed for approach and landing of aircraft. These new applications place strict requirements regarding position accuracy and system availability. In order to realize these requirements, it is essential to develop robust antenna designs. In this contribution, a multiband antenna array suitable for safety-of-life satellite navigation is presented. The array consists of 7 elements which are arranged on a half sphere. The single elements are resonant at the Galileo $\mathrm{E5a} / \mathrm{b}$ and E1 frequency bands. The geometry of the array allows it to achieve stable gain and axial ratio performance over the whole upper hemisphere.
\end{abstract}

\section{INTRODUCTION}

Antennas are the interface between the incoming navigation signal from the satellites and the receiver in a global navigation satellite system (GNSS). This important position that the antenna occupies lends it as a point of solution to several of the existing and emerging threats to dependable satellite navigation. One of such threats is multipath propagation which can distort the computed pseudoranges of the receiver from the satellites. This threat is particularly important when GNSS is utilized in terrestrial domains such as approach and landing of aircraft or for railways in mountainous terrain. To combat multipath, antennas with good axial ratios performance are desirable because of their ability to differentiate between the right hand circularly polarized (RHCP) direct satellite signals and the multipath signals which are predominantly of left hand circular (LHC) or elliptical polarization. Since satellite signals are very weak when they arrive at the receiver, it is also essential for the antenna to have a good gain pattern in the direction of the arriving signal i.e in the upper hemisphere. Both the gain and axial ratio performance of most antennas are not ideal at low elevations. Signal reception at low elevation is, however, important as it results in a better geometrical dilution of precision and can also improve the total system availability as more satellites become useable. These advantages have motivated research and development of antennas with wide angular coverage in terms of gain and axial ratio [1]-[3].

Apart from the aforementioned inherent threats to reliable satellite navigation, there are additional issues that are becoming important, for instance, the threat from radio-frequency interference (RFI) which can be accidental or intentional. Accidental interference can arise when personal privacy devices (PPDs) are active in the vicinity of the receiver. This situation is common near airports where interference arrives from nearby highways. In this case, the jammer has the goal of disabling its own navigation system usually to avoid being tracked. The interference from these devices can affect the aircraft receiving antenna as well as the augmentation system base station e.g. in ground-based augmentation systems (GBAS). Intentional interference is less frequent but is becoming more common. This kind of interference can result from the installation of jamming devices at certain locations in order to disrupt navigation services, e.g. against unmanned aerial vehicles (UAVs), at the particular location for security or privacy reasons. More malicious intentional interferences are those that seek to disrupt navigation services for criminal reasons such as at sea.

A more sophisticated and emerging class of external threat to satellite navigation comes from spoofers. A spoofer is an agent that transmit signals that resemble the ones from the satellites in order to gain control over the target's navigation system. Once the navigation system of the target is infiltrated, the spoofer can then control its movement with damaging consequences.

Recent research activities have focused on developing solutions for safety critical satellite navigation for multipath suppression, interference mitigation, protection against spoofers, enhancement of signal availability and to improve the precision of the obtained receiver position [4]-[11]. At the heart of most of the solutions is an antenna array because of the additional degrees of freedom that they provide. In [10], for instance, the direction of arrival of the jamming signal is detected by means of an antenna array and beamforming algorithms are utilized to place nulls towards the jammers and steer the beam of the antenna towards the satellite. For these applications, conformal arrays offer the possibility to increase the coverage angles at lower elevation as opposed to planar arrays whose performance degrade at lower elevation. These antennas take advantage of the shape of the carrier which can be naturally suited for omnidirectional coverage e.g. spherical or cylindrical shapes [12], [13].

In this contribution, a multi-band conformal antenna array is presented. The array consists of seven elements with one element at the top and the remaining six, radially directed elements, are arranged in a ring below the top element in a spherical cap. The shape of the array allows the array gain to 
be fairly constant over the whole forward hemisphere. Also, in order to enable the reception of multiple GNSS systems such as the upcoming European Galileo, the Russian GLONASS and the Chinese BeiDou systems, the antenna elements are resonant at multiple frequency bands.

\section{Element Design}

The single element consists of two probe-fed stacked patches. The dimensions of the patches are selected such that the lower patch, closer to the ground plane, resonates at the E5 frequency band and the upper patch resonates at the E1 frequency band. In order to excite both the vertical and horizontal current modes of the patches, two feeds are utilized at the shown locations. Circular polarization is obtained by introducing a phase shift of $90^{\circ}$ between the feeds by means of a commercial off-the-shelf hybrid coupler. In order to compensate for the probe inductance, the feeds are terminated by capacitive caps of radius $2.5 \mathrm{~mm}$. The layout of the single element antenna is shown in Fig. 1. A thicker layer of substrate
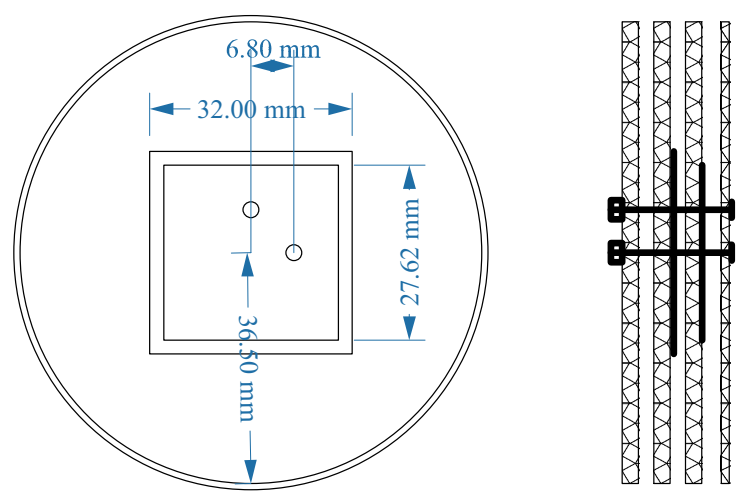

Fig. 1. Layout of the single antenna element. The upper dielectric layer has a thickness of $1.27 \mathrm{~mm}$ and the remaining three layers have a thickness of $2.54 \mathrm{~mm}$.

is required below the E5 resonant patch due to the larger bandwidth requirement for the $\mathrm{E} 5(50 \mathrm{MHz})$ frequency band compared to the $\mathrm{L} 1(32 \mathrm{MHz})$ resonant patch. The antenna utilizes a substrate with a dielectric constant of 10.20 . The dielectric layers are glued together with an adhesive material having a dielectric constant of 3.17 in order to ensure physical robustness of the antenna. The stack-up is then embedded in a circular cavity so as to ensure that the radiation characteristics of the antenna does not change drastically when it is embedded in the metallic holder of the array. The manufactured antenna is shown in Fig. 2. It was measured in a spherical nearfield chamber in order verify the radiation characteristics. The realized gain at zenith against frequency is shown in Fig. 3 and the far-field cuts are shown in Fig. 4 and Fig. 5 for the E5 and E1 central frequencies, respectively. It can be observed that the antenna achieves circular polarization within the desired frequency bands with a peak separation of more than $20 \mathrm{~dB}$ between the RHCP and LHCP gains.

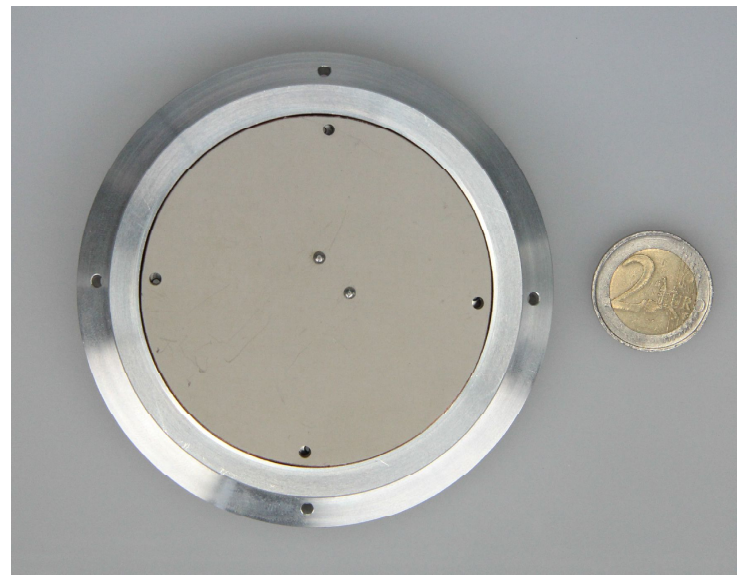

Fig. 2. Manufactured single element.

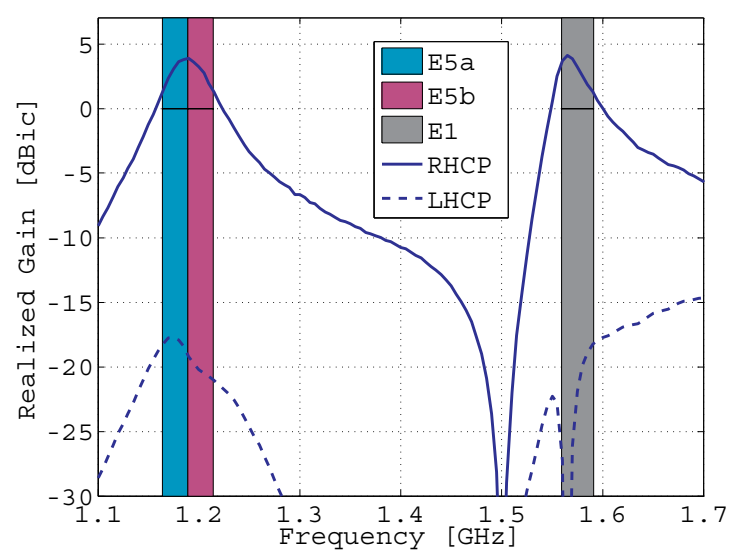

Fig. 3. Measured realized gain of the manufactured single element at boresight. The antenna was measured in the metallic holder shown in Fig. 2 as ground plane.

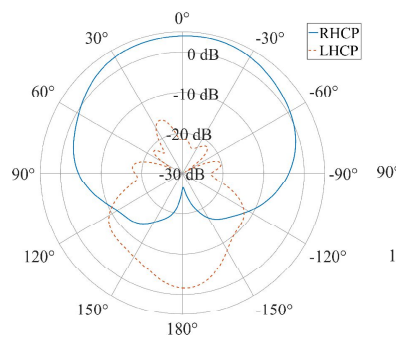

(a) $\mathrm{Phi}=0^{\circ}$

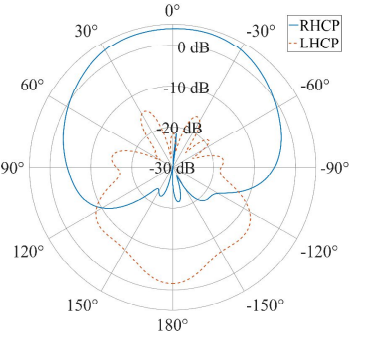

(b) $\mathrm{Phi}=90^{\circ}$
Fig. 4. Measured realized gain of the single antenna against theta angle at E5 central frequency of $1.189 \mathrm{GHz}$.

\section{ArRay Architecture}

Identical antenna elements are manufactured and arranged to form an array. The geometry of the array is similar to the simulated antenna in [5]. It consists of seven antenna elements distributed on a half sphere with one element on top of the sphere and the remaining six, radially directed, elements 


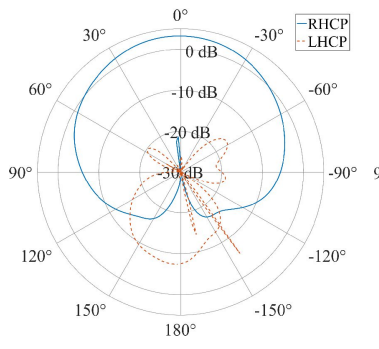

(a) $\mathrm{Phi}=0^{\circ}$

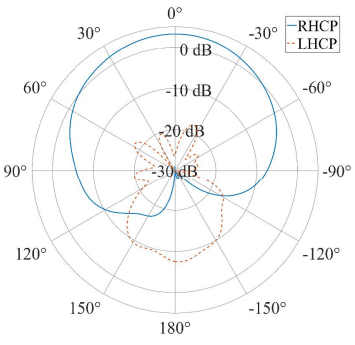

(b) Phi $=90^{\circ}$
Fig. 5. Measured realized gain of the single antenna against theta angle at E1 central frequency of $1.575 \mathrm{GHz}$.

arranged on a ring below the upper element as shown in Fig. 6. The equatorial diameter of the aluminum cap is $238 \mathrm{~mm}$. Each antenna element is connected to a low noise amplifier and RF filters so that adequate power can be delivered to the receiver (Fig. 7).

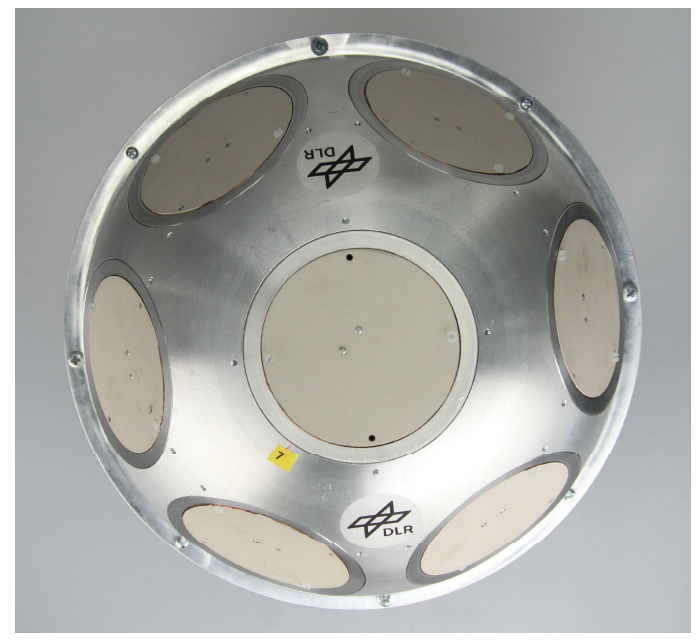

Fig. 6. The manufactured antenna array.

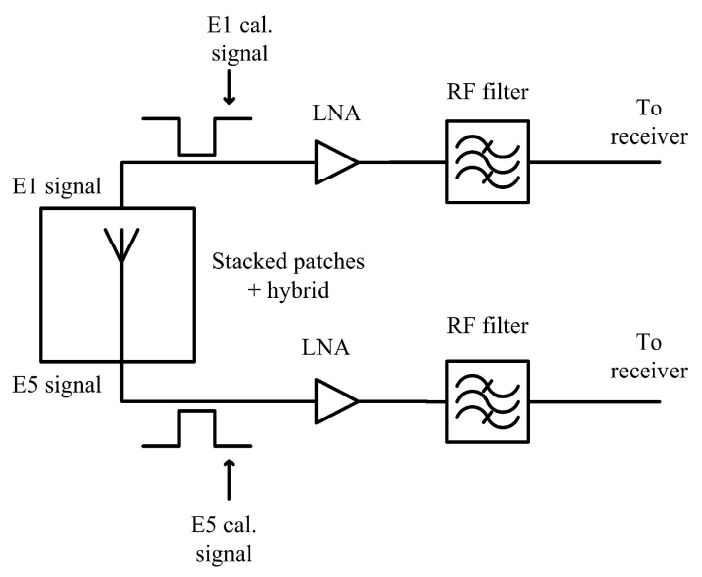

Fig. 7. Active antenna architecture.

\section{ArRay CALIBration}

To fully benefit from the possibilities that a multi-antenna navigation receiver can offer, it must be calibrated. The calibration should be performed for the whole signal paths of all antenna elements, including all electronic components, cables and analog-to-digital converters (ADC) of a digital receiver. The calibration accuracy has considerable influence on the achievable performance of the navigation receiver, e. g. ability to form deep nulls in the directions of interferers. Radiation characteristics of passive antenna elements do not change significantly over time and it is mostly enough to calibrate them once before putting the receiver into operation. However, active components of the active antenna architecture (Fig. 7) and front-ends can considerably change their characteristics due to temperature changes and aging process and therefore should be calibrated frequently. That is why a calibration concept similar to [14] was applied. In this concept, a calibration network is integrated into the antenna structure and distributes a calibration signal to all antenna outputs. This signal travels through the whole signal paths behind the antenna to a digital receiver and enables permanent on-line calibration. The calibration network for the conformal antenna is shown in Fig. 8. It is based on 1:2 power dividers with

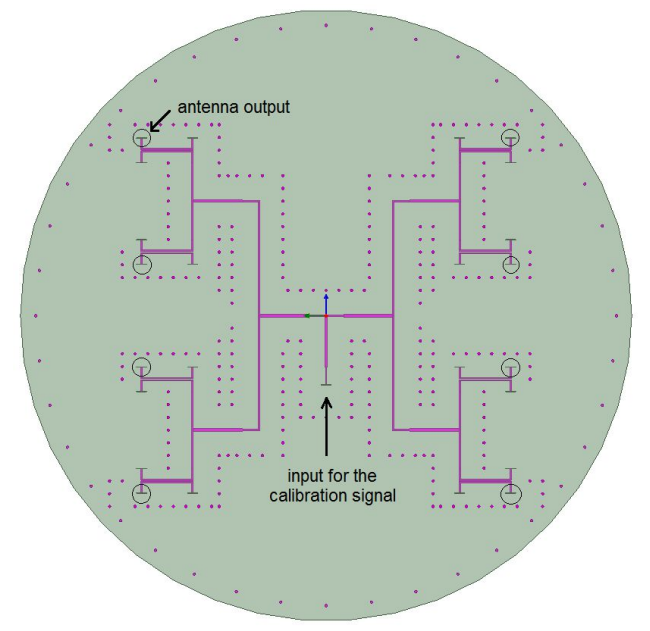

Fig. 8. Top view of the calibration network simulation model.

$\lambda / 4$ impedance transformers to deliver signals of the same amplitude and phase for each of the eight outputs. Directional couplers at each output couple the calibration signal to the antenna outputs. As the proposed conformal antenna has only seven elements, one of the outputs of the calibration network is terminated by $50 \Omega$. To enhance the stability of the calibration network it was designed in stripline technology with shielding vias. As a result very stable amplitude and phase transfer characteristics were obtained. Measured results revealed that amplitudes vary less than $0.15 \mathrm{~dB}$ in each of the frequency bands and the phases are highly linear with a difference between the outputs of less than $4^{\circ}$. Fig. 9 shows the measured phase transfer characteristics for all outputs of the calibration network. 


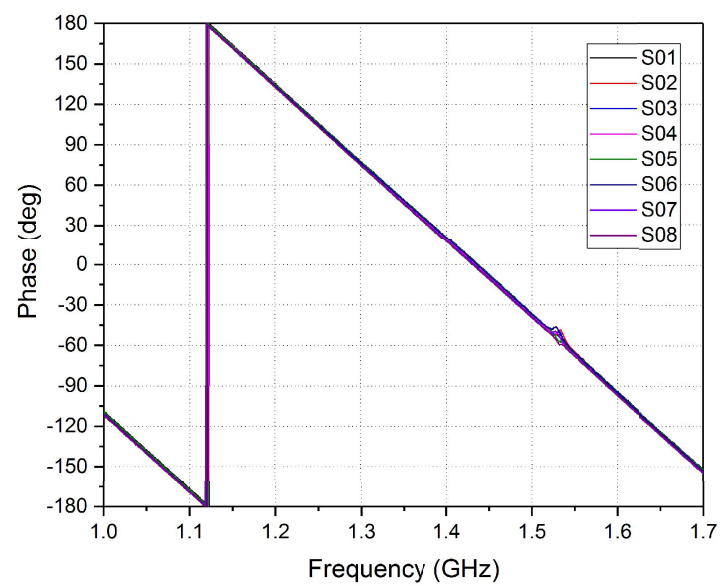

Fig. 9. Phase transfer characteristics of the calibration network.

\section{EMBEDdED FAR-Field PATTERnS}

The antenna was measured in an anechoic near-field chamber equipped with a spherical positioner. The embedded pattern of each antenna was measured while the remaining antenna ports in the array are terminated by $50 \Omega$ resistors. Fig. 10 and Fig. 11 show the embedded realized gain patterns of the antenna at $1.575 \mathrm{GHz}$ and at $1.189 \mathrm{GHz}$, respectively. It

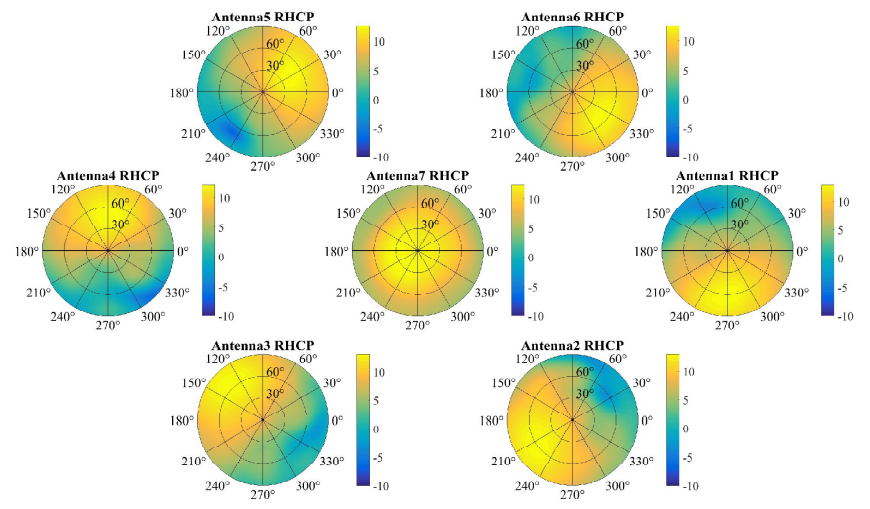

Fig. 10. Embedded RHCP gain patterns of the antenna elements at $1.575 \mathrm{GHz}$ in $\mathrm{dB}$.

is observed that, due to the geometry of the array, the antenna is able to offer a complete coverage of the upper hemisphere at the desired frequency bands.

\section{CONCLUSION}

A spherical cap conformal antenna array was presented. The array achieves good gain and axial ratio performance over the upper hemisphere and is resonant at multiple GNSS frequency bands. The array structure and its performance at low elevation could help to mitigate interference and multipath. The antenna can reduce the elevation mask of a GNSS receiver thereby increasing the number of satellites that can be used for positioning. Moreover, resonance at multiple frequency bands

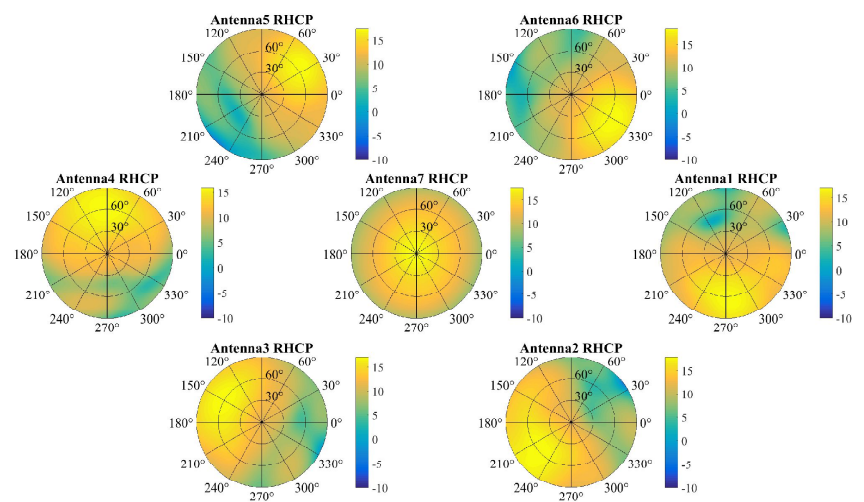

Fig. 11. Embedded RHCP gain patterns of the antenna elements at $1.189 \mathrm{GHz}$ in $\mathrm{dB}$.

can be useful for multi-constellation positioning and multifrequency ionospheric corrections.

\section{REFERENCES}

[1] K. M. Mak and K. M. Luk, "A circularly polarized antenna with wide axial ratio beamwidth," IEEE Transactions on Antennas and Propagation, vol. 57, no. 10, pp. 3309-3312, Oct 2009.

[2] L. Chen, T. L. Zhang, C. Wang, and X. W. Shi, "Wideband circularly polarized microstrip antenna with wide beamwidth," IEEE Antennas and Wireless Propagation Letters, vol. 13, pp. 1577-1580, 2014.

[3] Y. Luo, Q. X. Chu, and L. Zhu, "A low-profile wide-beamwidth circularly-polarized antenna via two pairs of parallel dipoles in a square contour," IEEE Transactions on Antennas and Propagation, vol. 63, no. 3, pp. 931-936, March 2015.

[4] S.-S. Hwang and J. J. Shynk, "Multicomponent receiver architectures for gps interference suppression," IEEE Transactions on Aerospace and Electronic Systems, vol. 42, no. 2, pp. 489-502, April 2006.

[5] J. Lambert, C. Balanis, and D. DeCarlo, "Spherical cap adaptive antennas for gps," Antennas and Propagation, IEEE Transactions on, vol. 57, no. 2, pp. 406-413, Feb 2009.

[6] M. V. T. Heckler, M. Cuntz, A. Konovaltsev, L. A. Greda, A. Dreher, and M. Meurer, "Development of robust safety-of-life navigation receivers," IEEE Transactions on Microwave Theory and Techniques, vol. 59, no. 4, pp. 998-1005, April 2011.

[7] C. J. Wullems, "A spoofing detection method for civilian $11 \mathrm{gps}$ and the e1-b galileo safety of life service," IEEE Transactions on Aerospace and Electronic Systems, vol. 48, no. 4, pp. 2849-2864, October 2012.

[8] L. Heng, D. B. Work, and G. X. Gao, "Gps signal authentication from cooperative peers," IEEE Transactions on Intelligent Transportation Systems, vol. 16, no. 4, pp. 1794-1805, Aug 2015.

[9] M. L. Psiaki, T. E. Humphreys, and B. Stauffer, "Attackers can spoof navigation signals without our knowledge. here's how to fight back gps lies," IEEE Spectrum, vol. 53, no. 8, pp. 26-53, August 2016.

[10] M. Cuntz, A. Konovaltsev, and M. Meurer, "Concepts, development, and validation of multiantenna gnss receivers for resilient navigation," Proceedings of the IEEE, vol. 104, no. 6, pp. 1288-1301, June 2016.

[11] K. A. Yinusa, W. Elmarissi, S. Caizzone, and A. Dreher, "A multiband conformal antenna array for gnss applications," in 2016 IEEE International Symposium on Antennas and Propagation (APSURSI), June 2016, pp. 897-898.

[12] S. Raffaelli, Z. Sipus, and P. S. Kildal, "Analysis and measurements of conformal patch array antennas on multilayer circular cylinder," IEEE Transactions on Antennas and Propagation, vol. 53, no. 3, pp. 11051113, March 2005.

[13] L. Josefsson and P. Persson, Conformal Array Antenna Theory and Design. Piscataway, NJ: IEEE Press, 2006.

[14] A. Konovaltsev, M. Cuntz, L. A. Greda, M. V. T. Heckler, and M. Meurer, "Antenna and rf front end calibration in a gnss array receiver," in 2010 IEEE International Microwave Workshop Series on RF Front-ends for Software Defined and Cognitive Radio Solutions (IMWS), Feb 2010, pp. 1-4. 